\title{
Diseño estructural de un stent endovascular para el tratamiento de un aneurisma aórtico ${ }^{1}$
}

\author{
Structural design of an endovascular stent for treatment of an aortic \\ aneurysm
}

\section{Desenho estrutural de um stent endovascular para o tratamento de um aneurisma da aorta}

\author{
S. Aristizábal, G. Suárez y J. S. Parra \\ Recibido: septiembre 13 de 2017- Aceptado: junio 30 de 2018
}

\begin{abstract}
Resumen - Los actuales modelos de stents endovasculares tienen un biomecanismo auto-expandible con memoria de forma del material, lo que en principio pretenden es un ajuste adecuado con el medio de implantación arterial. Sin embargo, presentan algunos problemas de desacoplamiento y de implantación en la arteria por diversas variabilidades biológicas. Como una alternativa a estas situaciones, se desarrolló un modelo de stent aórtico regulable (ajustable). Este modelo de movilidad controlada pretende facilitar el procedimiento de implantación y el no desacoplamiento por la actividad hemodinámica. Se elaboró un diseño geométrico y se desarrollaron diversas simulaciones para analizar su comportamiento biomecánico utilizando como herramienta computacional el programa Working Model. Se obtuvieron unos resultados que indicaron la viabilidad y correcta funcionalidad de esta alternativa para el problema del desacople e implantación.
\end{abstract}

${ }^{1}$ Producto derivado del proyecto de investigación "Simulación de una bomba de asistencia ventricular cardiaca en la interface fluido mecánico como problema acoplado", del Grupo de Investigación en Matemáticas y el Grupo de Investigación en Dinámica cardiovascular de la Universidad Pontificia Bolivariana.

S. Aristizábal, Universidad Pontificia Bolivariana, Medellín (Colombia).

G. Suárez, Universidad Pontificia Bolivariana, Medellín, Colombia, email: gustavo.suarez@upb.edu.co.

J. S. Parra, Universidad Pontificia Bolivariana, Medellín, Colombia. email: juans.parra@upb.edu.co.

Como citar este artículo: Aristizábal, S., Suárez, G. y Parra, S. Diseño estructural de un stent endovascular para el tratamiento de un aneurisma aórtico. Entre Ciencia e Ingeniería, vol. 12, no. 24, pp.9-14, julio-diciembre, 2018. DOI: http://dx.doi.org/10.31908/19098367.3809

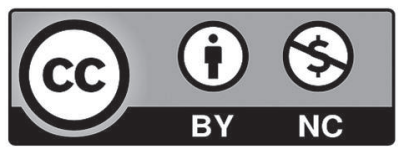

Attribution-NonCommercial 4.0 International (CC BY-NC 4.0)
Palabras clave - aneurisma, mecanismo ajustable, Stent endovascular, working model.

Abstract - The current models of endovascular stents have an auto-expanding biomechanism with shape memory material which is able to adjust itself in the middle of implantation. These models have problems in implantation procedures in the artery; including problems of decoupling. As an alternative to the situation, a new aortic adjustable stent was developed. This model of controlled mobility aims to facilitate the implantation procedure and the non-decoupling due to the hemodynamic activity. A geometric design was developed, and several simulations were developed to analyze its biomechanical behavior using the Working Model program as a computational tool. Some results were obtained that indicated the feasibility and correct functionality of this alternative for the problem of decoupling and implantation.

Keywords- aneurysm endovascular Stent, adjustable, aneurysm, aortic, working model.

Resumo- Os modelos atuais de stents endovasculares têm um biomecanismo auto-expansível com memória de forma do material, que em princípio se destina a ser um ajuste adequado com os meios de implantação arterial. No entanto, eles apresentam alguns problemas de desacoplamento e implantação na artéria devido a várias variabilidades biológicas. Como alternativa a estas situações, foi desenvolvido um modelo de stent aórtico ajustável. Este modelo de mobilidade controlada visa facilitar o procedimento de implantação e o não-desacoplamento devido à atividade hemodinâmica. Um projeto geométrico foi desenvolvido e diversas simulações foram desenvolvidas para analisar seu comportamento biomecânico utilizando o programa Working Model como ferramenta computacional. Foram obtidos alguns resultados que indicaram a viabilidade e correta funcionalidade desta alternativa para o problema de desacoplamento e implantação.

Palavras chave - aneurisma, endoprótese endovascular, mecanismo ajustável, modelo de trabalho. 


\section{INTRODUCCIÓN}

A nivel mundial, las tasas de morbilidad y mortalidad asociadas a aneurismas aórticos vienen en crecimiento debido al aumento de enfermedades causadas por la hipertensión, la arteriosclerosis y el tabaquismo [1], [2].

Los aneurismas de aorta abdominal (AAA) son una patología potencialmente mortal presente entre el 2 y $4 \%$ de la población mayor de 50 años, y tiene asociada una mortalidad entre el 0,7 y 5\%. Los aneurismas de aorta torácica tienen asociada una mortalidad entre el 4 y $12 \%$, y en el caso de rotura, entre el 19 y $70 \%$ [1], [2].

Por estas razones, las prótesis endovasculares son un tema de gran interés investigativo, pues resulta evidente el aumento significativo en cuanto al número de pacientes enfermos por patologías cardiacas, así como las múltiples limitaciones y dificultades que presentan los modelos actuales de stents [2]-[4].

Los problemas asociados al desacoplamiento del stent una vez implantado en el paciente enfermo, se presentan debido a la variabilidad del diámetro de la región arterial afectada, ya que por comportamientos biológicos naturales esta área cambia su forma, incidiendo en las dimensiones predeterminadas del diseño del prototipo (situación que genera endofugas o endoleak).

Dicho problema se ha observado entre el 20 y $25 \%$ de los pacientes tratados, y se manifiesta por el mal funcionamiento de la prótesis y por las alteraciones del flujo en la actividad hemodinámica posterior a la implantación [2]-[5].

Como alternativa desarrollada, se diseñó un stent con movilidad auto-ajustable, el cual posee una dinámica física independiente sobre cualquier variación del área de contacto donde se instale el implante protésico; además, con dimensiones apropiadas para la inserción a través de la arteria femoral y la implantación en el vaso aórtico.

\section{ANTECEDENTES Y ASPECTOS TÉCNICOS}

Son diversos los grupos de investigación en bioingeniería y medicina que vienen orientando sus estudios en la búsqueda de soluciones adecuadas para los diferentes casos de patologías cardiacas [3], [9]-[11]. Algunos de ellos buscan mejorar los actuales diseños de stents endovasculares, perfeccionar los procedimientos de implantación y adecuación de la región afectada, entre otras situaciones clínicas que se presentan [6]-[8], [13].

Distintos estudios clínicos manifiestan que la mayor problemática se presenta en el diseño geométrico del stent, debido a problemas de: embolización, oclusión y filtración producidas en los procedimientos de inserción e implantación [4],[10], [11], [13].

Otras investigaciones sobre tipos de aneurismas y problemas de arterosclerosis, señalan que es fundamental determinar el tipo de daño vascular para poder delimitar en cada caso, cuál sería la mejor solución y uso adecuado del stent endovascular [4], [12]-[14], como se ilustra en la Fig.1.

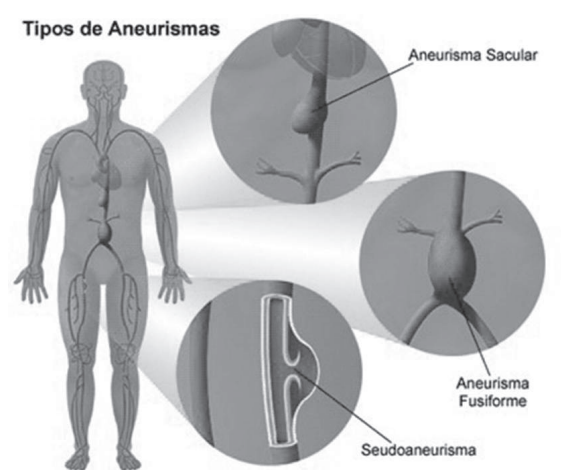

Fig. 1. Casos clínicos de aneurismas sacular, fusiforme, seudo-aneurismas [21].

\section{Desarrollo}

\section{A. Materiales y métodos}

Para el desarrollo de la investigación, fue importante determinar el tipo de aneurisma que se deseaba tratar, cuya descripción se observa en la Tabla 1.

TABLA I

DIFICULTADES DE LAS PRÓTESIS ENDOVASCULARES CONSIDERADAS EN EL DISEÑO DEL MODELO

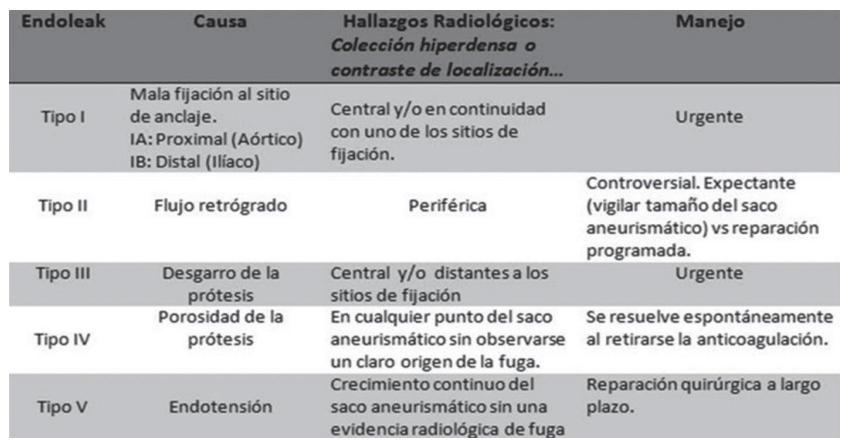

Con base en varios estudios estadísticos, se evaluó que el tipo I es el que registra mayores incidentes de problemas en pacientes enfermos. Este caso hace mención a:

Tipo I, mala fijación al sitio de anclaje sea IA proximal (Aórtico) o IB Distal (Ilíaco), y se produce cuando hay una fuga de sangre al saco aneurismático a través de uno de los sitios de fijación de la prótesis. Por tanto, esta patología se subdivide de acuerdo con la localización de la fuga en Tipo $1^{\mathrm{a}}$, cuando es proximal (extremo aórtico), y Tipo 1B si es distal (extremo ilíaco).

En cualquiera de los dos tipos se produce una separación entre las prótesis y la pared arterial nativa, creando una comunicación directa entre el saco aneurismático y la circulación arterial sistémica [4].

\section{B. Parámetros y variables del sistema}

Para el diseño del stent aórtico regulable (ajustable) se consideró un diámetro máximo de apertura de $3,2 \mathrm{~cm}$, con un diámetro de retracción de $1,18 \mathrm{~cm}$, lo que permite el tránsito del stent por todo el sistema arterial hasta el lugar donde será implantado. El stent es transportado a través de la arteria aorta descendente, la cual presenta un diámetro aprox. de 1,6 cm; la arteria aorta abdominal, que presenta un diámetro aprox. 
de $3 \mathrm{~cm}$ y la arteria femoral, que tiene un diámetro aprox. de $1,8 \mathrm{~cm}$ [19], [20].

El material utilizado para la elaboración del stent fue un polímero poliacetal debido a sus propiedades biocompatibles, flexibilidad mecánica y operabilidad [24].

Algunas de las investigaciones sobre tratados en diseños de stents han considerado condiciones biofísicas y propiedades mecánicas referentes a tensión del material, resistencia mecánica a la fatiga, resistencia mecánica radial y análisis de expansión-retracción, esto debido principalmente a que los diseños actuales tienen memoria de forma, situación por la cual se encuentran sometidos a condiciones de cargas [15]- [18].

En el diseño desarrollado, estos parámetros y variables no fueron considerados por las siguientes razones: el modelo después de implementado queda en una posición estable y rígida, por lo que su movilidad se da solo en la instalación no generándose efectos de tensión permanente. Además, no se requirió un análisis de fatiga ya que el modelo estará en un estado de fijación permanente inmóvil, cuya única función será la de mantener abierta la aorta para el tránsito del flujo sanguíneo.

La presión del flujo sanguíneo en la arteria aórtica es aproximadamente de $100 \mathrm{mmHg}$, condición biofísica que no ejerce una fuerza significativa en el stent elaborado con material poliacetal, que tiene una resistencia a la ruptura por compresión igual a $110 \mathrm{MPa}$ [24].

Una particularidad del stent diseñado es la posibilidad de expandirse y retraerse mecánicamente, controlado por elementos deslizadores que no generan fuerzas resistivas.

\section{Metodología}

Se elaboró un esquema de la metodología para el desarrollo de la investigación, la cual se presenta a continuación en la Fig. 2.

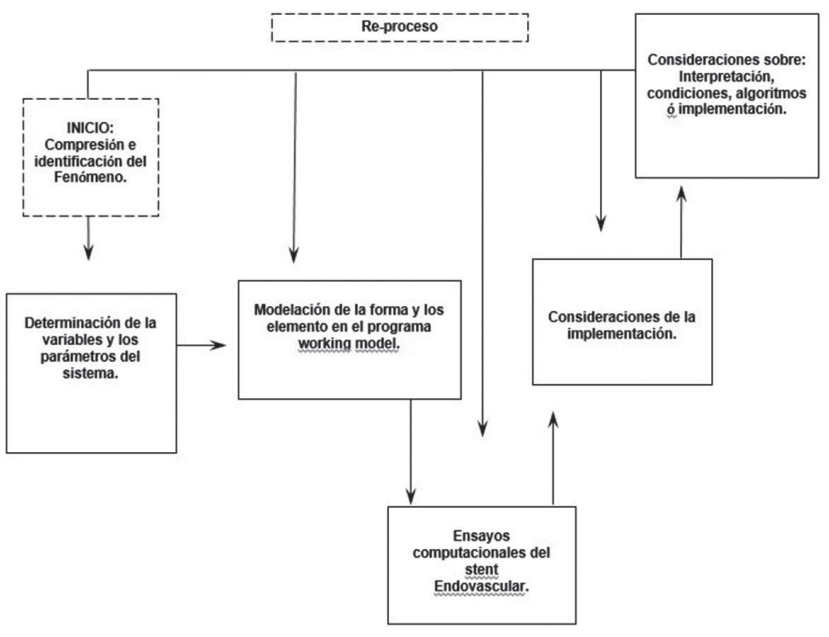

Fig. 2. Metodología para la elaboración del stent endovacular.

\section{ANÁLISIS Y RESULTADOS}

\section{A. Modelos de stent endovascular diseñados}

Se diseñaron diversos modelos de stent endovascular con un sistema regulable (ajustable), ya que dicha condición permite acomodar el modelo de manera perfecta en regiones arteriales de superficies irregulares, con el fin de evitar el desacoplamiento generado por la actividad del flujo sanguíneo, como se muestra en la Fig. 3.

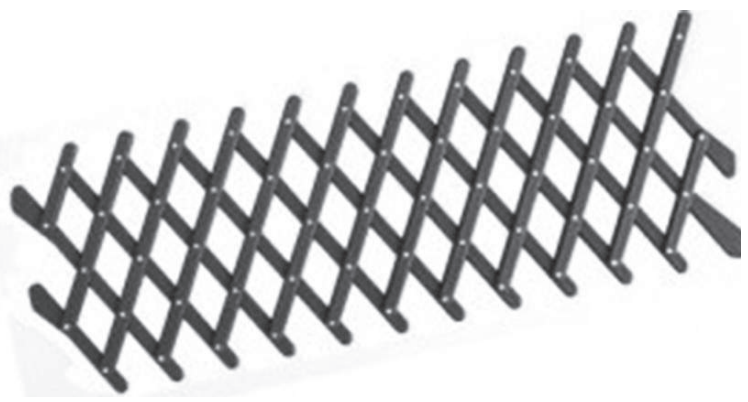

Fig. 3. Sistema movible mecánico ajustable [22].

Las dimensiones que poseen los stent de tamaño milimétrico es un factor dimensional que complejizó la labor de obtención de un modelo que brindara apropiada funcionalidad y desempeño dentro de la arteria afectada, lo que se puede observar en la Fig. 4.

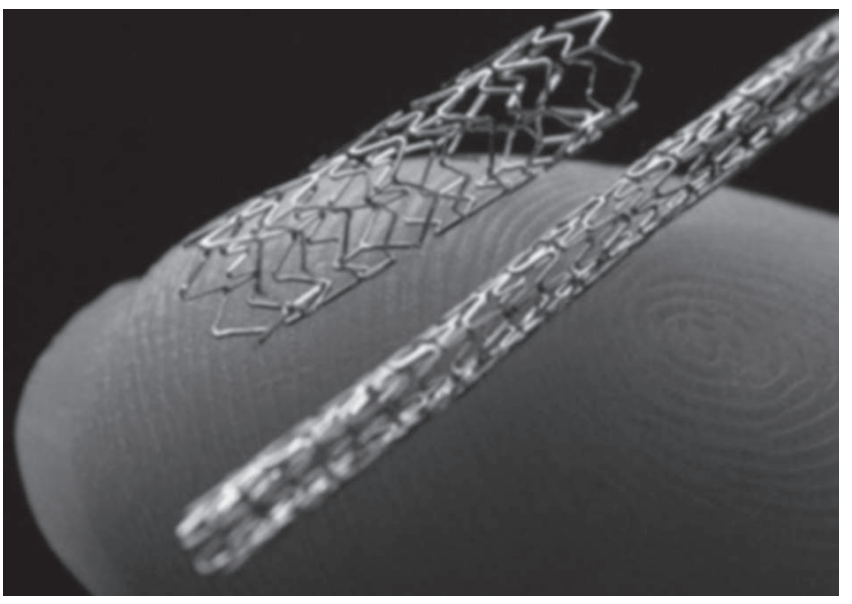

Fig. 4. Tamaño milimétrico de los modelos de stents endovasculares [23].

Durante el proceso se elaboraron diversos análisis computacionales buscando diseños con mayor estabilidad (determinada al momento de abrir el stent de manera controlada y uniforme para evitar complicaciones en la implantación) en la estructura y una mayor área de contacto (puntos o superficies del stent que entran en contacto con la superficie del vaso arterial), con el propósito de asegurar mejor adaptabilidad a la superficie, ajuste y facilidad de implantación.

A continuación se describen los modelos de stent construidos dentro del proceso de diseño desarrollado. El modelo denominado M1 es uno de los primeros prototipos, el cual fue elaborado mediante la forma de un polígono de 9 lados y 9 segmentos, según Fig. 5 y Fig. 6. 


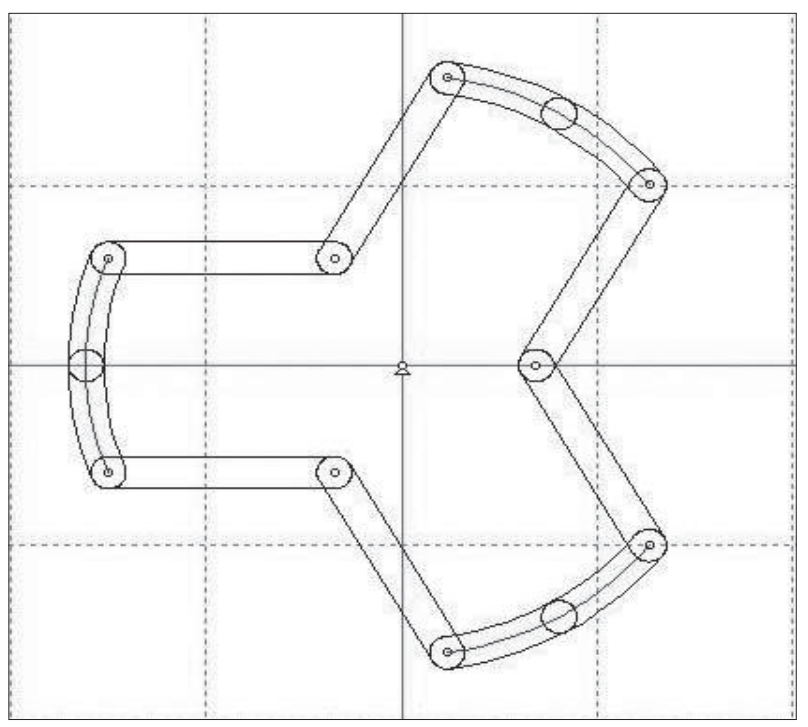

Fig. 5. Diseño de modelo M1 de stent endovascular (parcialmente cerrado) para tratamiento de aneurismas.

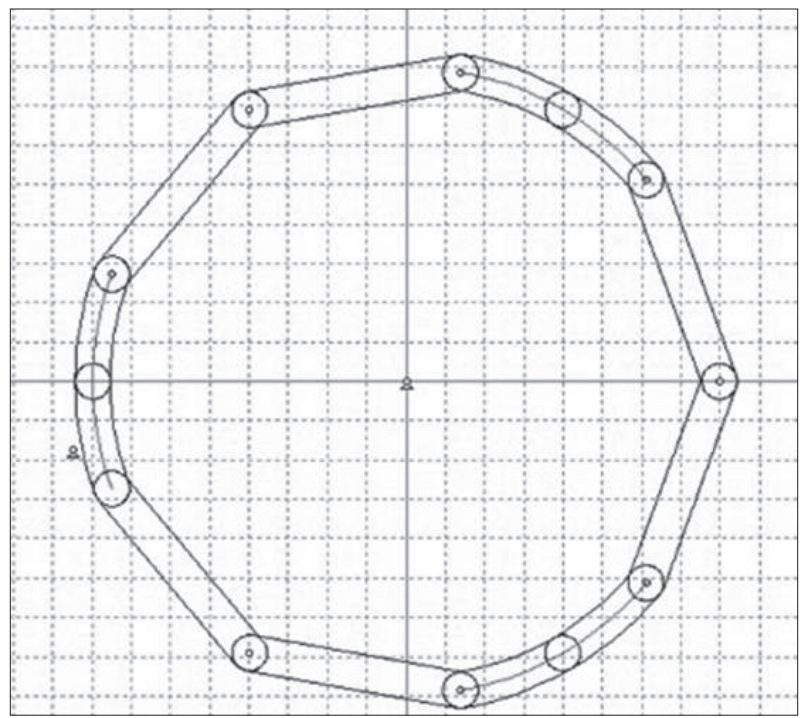

Fig. 6. Diseño de modelo M1 de stent endovascular (totalmente expandido) para tratamiento de aneurismas.

El modelo M1 presenta un buen desempeño respecto al área de contacto (tres palancas de nueve están en contacto con la superficie) y asegura un buen acople al vaso sanguíneo (articulaciones de palancas con la superficie), sin embargo, fue ineficaz la expansión del modelo una vez localizado en el lugar donde se desarrollaría la apertura (se genera un bloqueo de las palancas), en la apertura, situación que traería dificultades en la implantación, como se ve en la Fig. 7.

También se diseñó un modelo denominado M2 con base en la forma de un polígono de 9 lados y 24 segmentos arqueados, el cual presentó una inadecuada estabilidad (expansión no controlada palancas del modelo que no estuvieran sometidas a movilidad permanente, sino que se mantuvieran estables); sin embargo, se pudo hacer una mejora en el diseño modificando los segmentos (palancas) en arco por segmentos lineales, con lo que se aumentaría la estabilidad del diseño, como se observa en la Fig. 8.

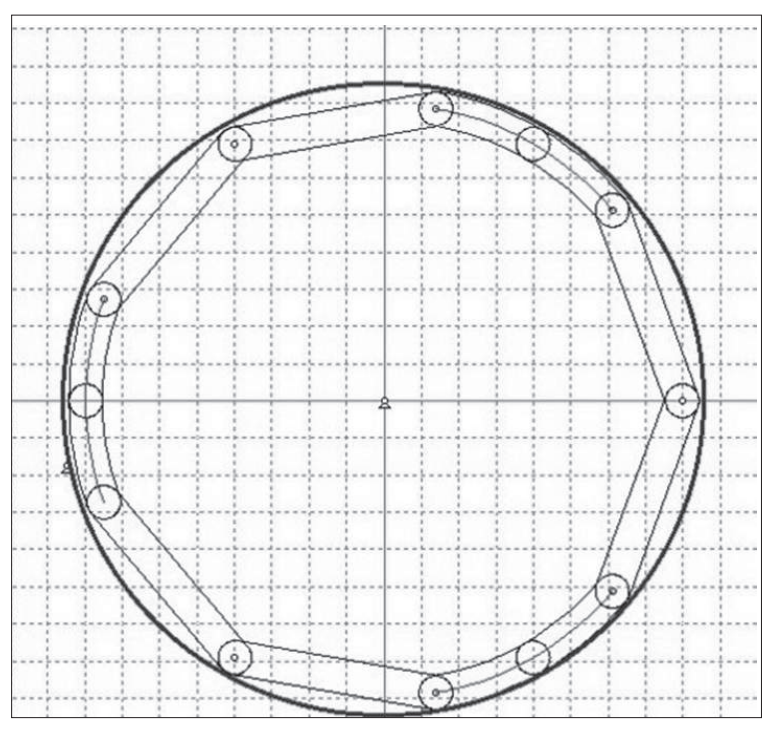

Fig. 7. Diseño de modelo M1 de stent endovascular enmarcado con la circunferencia como área de contacto arterial.

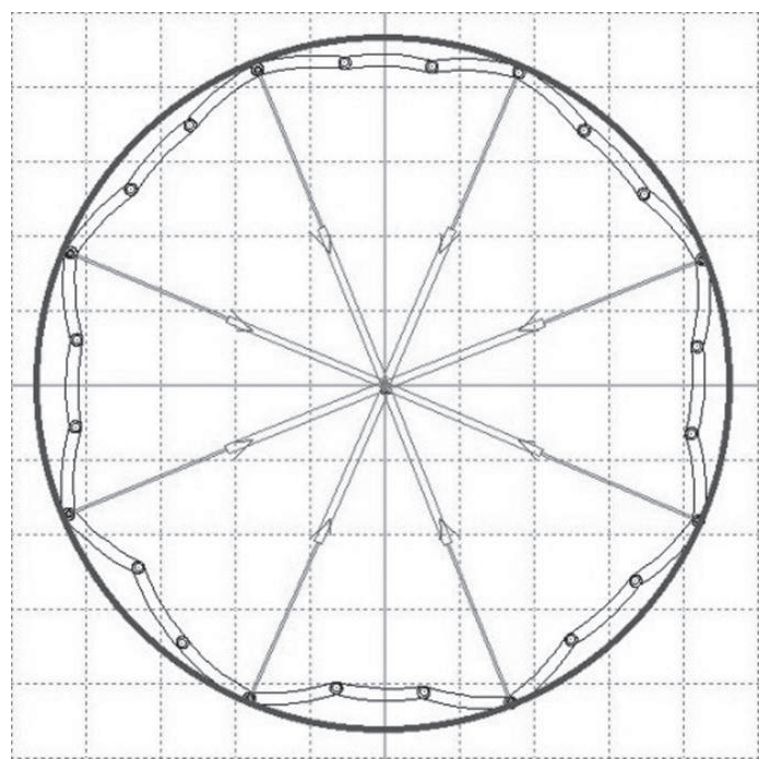

Fig. 8. Diseño de modelo M2 de stent endovascular enmarcado con la circunferencia como área de contacto arterial.

Como modelo definitivo denominado M3, se obtuvo el diseño mejorado de stent endovascular considerando las diferentes experiencias y rediseños computaciones. Así, se llegó al diseño en forma de un polígono de 9 lados y 24 segmentos que presentó una adecuada adaptabilidad, determinada por una apertura uniforme, sin bloqueo estructural, de fácil implantación y acoplada a la superficie por medio de 8 puntos en las uniones del stent, como se ve en la Fig. 9.

Dentro de los aspectos de diseño considerados, se enfatizó en los detalles del proceso de instalación física del stent hasta la arteria aortica, pasando a través de la arteria femoral, donde se podrían presentar algunos problemas de embolización visceral.

B. Evaluación y análisis del comportamiento biomecánico del modelo definitivo M3

Se obtuvo un modelo de stent de dimensión en apertura 


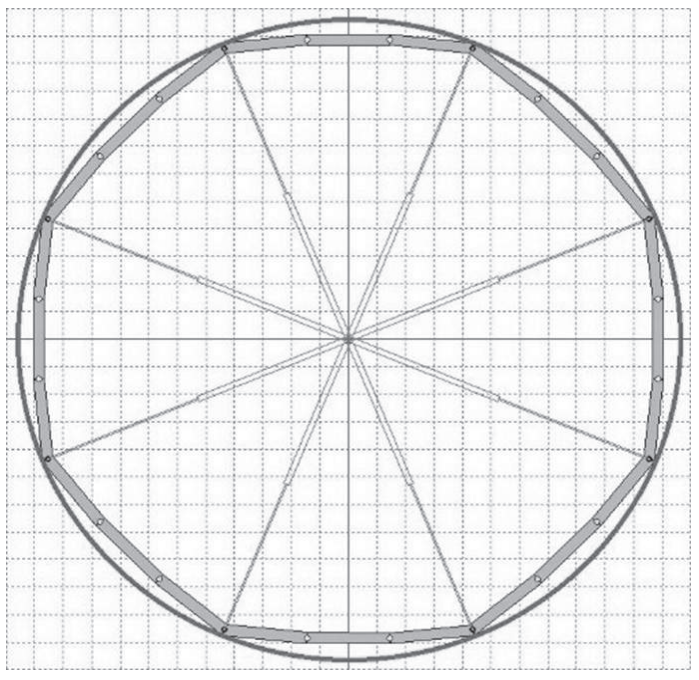

Fig. 9. Diseño del modelo stent endovascular M3, enmarcado con la circunferencia como área de contacto arterial.

$3,2 \mathrm{~cm}$ que se expande homogéneamente, se acopla adecuadamente al vaso sanguíneo a partir de 8 puntos del modelo (articulaciones), no presenta en la implantación colisiones de los mecanismos, no presenta dificultades de transporte del stent por la arteria femoral y se puede expandir máximo 2,75 veces su tamaño inicial, lo cual se muestra en Fig. 10, Fig. 11 y Fig. 12.

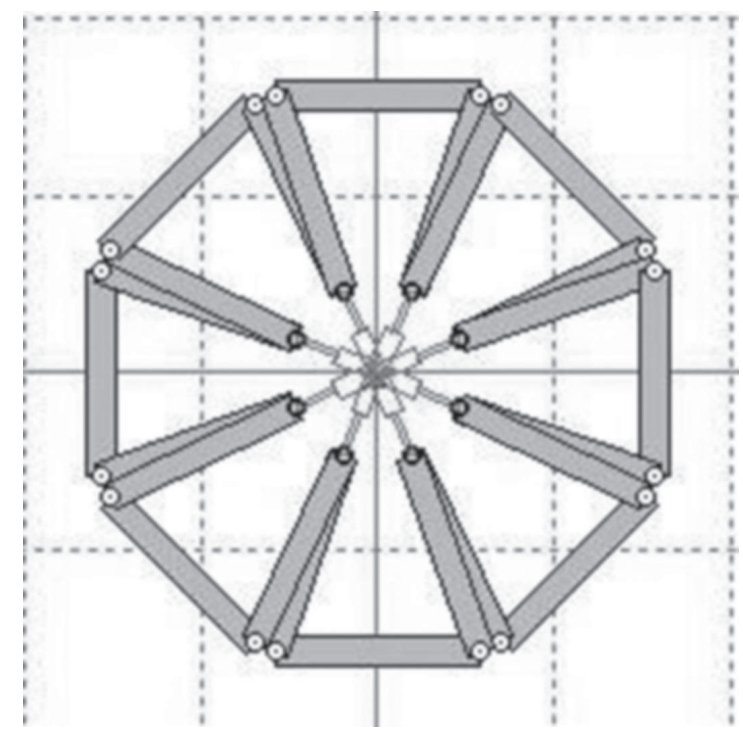

Fig. 10. Diseño modelo definitivo M3 de stent endovascular totalmente cerrado.

\section{Conclusiones}

Los diámetros de expansión de los modelos de stent desarrollados en esta investigación no están sujetos a la memoria de forma determinada por las propiedades y leyes constitutivas de los materiales, proporcionando así una mayor vida útil del modelo implantado, ya que los elementos de la estructura no sufren proceso de fatiga.

En el diseño del stent las propiedades de los materiales no son factor relevante, como en otros diseños existentes

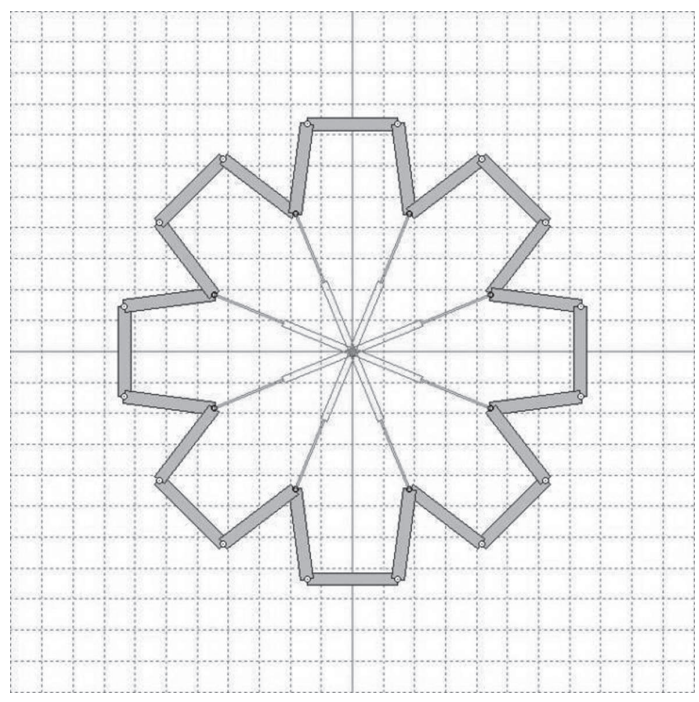

Fig. 11. Diseño modelo definitivo M3 de stent endovascular parcialmente abierto con una apertura controlada uniforme.

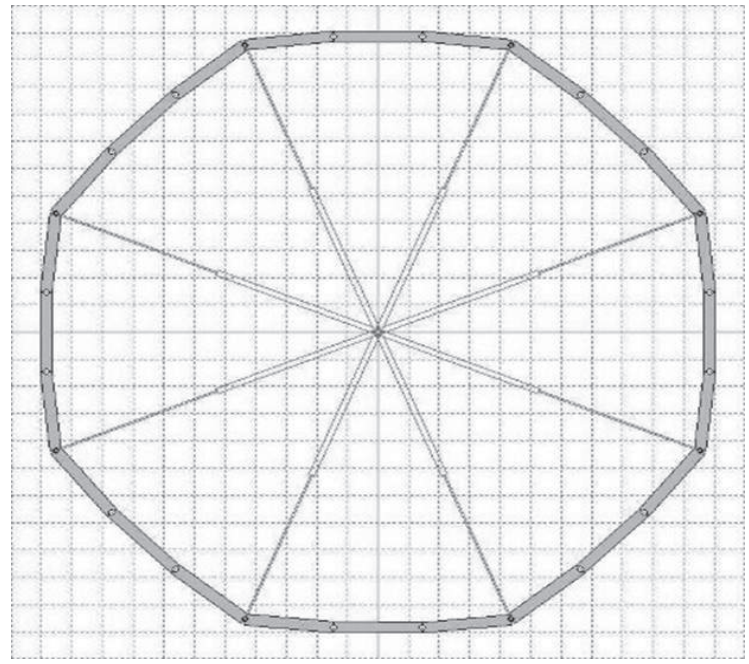

Fig. 12. Diseño modelo definitivo M3 de stent endovascular, totalmente expandido.

que involucran expansión por memoria de forma. Esta condición especial elaborada permite darle mayor relevancia a la biocompatibilidad con el cuerpo, desestimar la causa de fractura del prototipo y también asegurar un mejor acople en la arteria.

Las dimensiones del stent desarrollado se ajustan de acuerdo con las dimensiones de la arteria afectada, garantizando así su buena funcionalidad. Las dimensiones del stent cerrado son de $1,18 \mathrm{~cm}$ con posibilidad de expansión hasta de $3,2 \mathrm{~cm}$, lo que favorece cualquier cambio súbito de la superficie arterial.

Finalmente, se cumplió con requerimientos de instalación de la endoprótesis donde la arteria femoral tiene un diámetro de aprox. $1,8 \mathrm{~cm}$, y el stent cerrado para su traslado posee un diámetro de $1,18 \mathrm{~cm}$, garantizando el transporte y la inserción por la arteria femoral hasta el acople con la arteria aortica, y evitando dificultades de tipo embolización visceral.

\section{REFERENCIAS}

[1] Kent, A. C., Zwolak, R. M., Egorova, N. N., Riles, T. S., Manganaro, 
A., Moskowitz, A. J., Gelijns, A. C., and Greco, G. "Analysis of risk factors for abdominal aortic aneurysm in a cohort of more than 3 million individuals," J. Vasc. Surg., vol. 52, no. 3, pp. 539-548, 2010.

[2] Salazar S., M. E., Carballo F., C., Romero C., F., Giraldo M., J. P., Arias M., Y. and Abu-Suboh A., A. Endofugas o Endoleaks en Prótesis Endovasculares de Aneurismas de Aorta Abdominal: Lo que el Radiólogo debe saber en relación al Diagnóstico, Caracterización y Principios Básicos de Manejo. Congreso SERAM 2012.DOI: http:// dx.doi.org/10.1594/seram2012/S-1294

[3] Serra P., A., Guardiola, F. M. and Montilla, B. V. "Novedades en stents farmacoactivos. Actualización y futuros desarrollos," Rev. Española Cardiol. Supl., vol. 10, no. SUPPL. 3, p. 2C-11C, Jan. 2010.

[4] Mertens, R., Valdés, F. and Krämer, A. "Tratamiento Endovascular del Aneurisma de Aorta," Revista Medica de Chile vol. 131, no. 6 1, pp. 3-11, 2003. dx.doi.org/10.4067/S0034-98872003000600005

[5] Hsiao, H. M., Chiu, Y. H., Lee, K. H. and Lin, C. H. "Computational modeling of effects of intravascular stent design on key mechanical and hemodynamic behavior," CAD Comput. Aided Des., vol. 44, no. 8, pp. 757-765, 2012.

[6] Cambria, R.P., Brewster, D. C., Lauterbach, S. R., Kaufman, J. L., Geller, S., Fan, C.M., Greenfield, A., Hilgenberg, A.and Clouse, W. D. "Evolving experience with thoracic aortic stent graft repair," J. Vasc. Surg., vol. 35, no. 6, pp. 1129-1136, 2002.

[7] Cox, G. S., O'Hara, P. J., Hertzer, N. R., Piedmonte, M. R., Krajewski, L. P. and Beven, E. G. "Thoracoabdominal aneurysm repair: A representative experience," J. Vasc. Surg., vol. 15, no. 5, pp. 780-788, 1992.

[8] Stanley C., E., Crawford, J. L., Safi, H. J., Coselli, J. S., Hess, K. R., Brooks, B., Norton, H. J. and Glaeser, D. H. "Thoracoabdominal aortic aneurysms: Preoperative and intraoperative factors determining immediate and long-term results of operations in 605 patients," $J$. Vasc. Surg., vol. 3, no. 3, pp. 389-404, Mar. 1986.

[9] Dake, M. D., Miller, D. C., Mitchell, R. S., Semba, C. P., Moore, K. A. and Sakai, T. "The 'first generation' of endovascular stent-grafts for patients with aneurysms of the descending thoracic aorta," $J$. Thorac. Cardiovasc. Surg., vol. 116, no. 5, pp. 689-704, 1998.

[10] Dumoulin, C. and Cochelin, B. "Mechanical behaviour modelling of balloon-expandable stents," J. Biomech., vol. 33, no. 11, pp. 14611470, 2000.

[11] Greenberg, R., Resch, T., Nyman, U., Lindh, M., Brunkwall, J., Brunkwall, P., Malina, M., Koul, B., Lindblad, B. and Ivancev, K. "Endovascular repair of descending thoracic aortic aneurysms: an early experience with intermediate-term follow-up," J. Vasc. Surg., vol. 31, no. 1, pp. 147-156, 2000.

[12] Parodi, J. C., Palmaz, J. C. and Barone, H. D. "Transfemoral Intraluminal Graft Implantation for Abdominal Aortic Aneurysms," Ann. Vasc. Surg., vol. 5, no. 6, pp. 491-499, Nov. 1991.

[13] Semba, C. P., Kato, N., Kee, S.T., Lee, G. K., Mitchell, R. S., Miller, D. C. and Dake, M. D. "Acute Rupture of the Descending Thoracic Aorta: Repair with Use of Endovascular Stent-Grafts," J. Vasc. Interv. Radiol., vol. 8, no. 3, pp. 337-342, May 1997.

[14] Thompson, C. S., Gaxotte, V. D., Rodríguez, J. A., Ramaiah, V. G., Vranic, M., Ravi, R., DiMugno, L., Shafique, S., Olsen, D. and Diethrich, E. B. "Endoluminal stent grafting of the thoracic aorta: Initial experience with the Gore Excluder," J. Vasc. Surg., vol. 35, no. 6, pp. 1163-1170, 2002.

[15] De Bock, S., Iannaccone, F., De Santis, G., De Beule, M, Mortier, P., Verhegghe, B. and Segers, P. "Our capricious vessels: The influence of stent design and vessel geometry on the mechanics of intracranial aneurysm stent deployment," J. Biomech., vol. 45, no. 8, pp. $1353-$ 1359, 2012.

[16] Kumar, G. P., Cui, F., Phang, H. Q., Su, B., Leo, H. L. and Hon, J. K. F. "Design considerations and quantitative assessment for the development of percutaneous mitral valve stent," Med. Eng. Phys., vol. 36, no. 7, pp. 882-888, 2014.

[17] Azaouzi, M., Makradi, M. and Belouettar, S. "Numerical investigations of the structural behavior of a balloon expandable stent design using finite element method," Comput. Mater. Sci., vol. 72, pp. 54-61, 2013.

[18] Hara, H., Nakamura, M., Palmaz, J. C. and Schwartz, R. S. "Role of stent design and coatings on restenosis and thrombosis," Adv. Drug Deliv. Rev., vol. 58, no. 3, pp. 377-386, 2006.

[19] Erbel, R. and Eggebrecht, H. "Aortic dimensions and the risk of dissection.," Heart, vol. 92, no. 1, pp. 137-42, Jan. 2006

[20] Sandgren, T., Sonesson, B., Ahlgren, A. R. and Länne, T. "The diameter of the common femoral artery in healthy human: Influence of sex, age, and body size," J. Vasc. Surg., vol. 29, no. 3, pp. 503-510, 1999.

[21] Bernardi C. “Angiología e cirurgía vascular”. [En línea]. Disponible en: https://cirurgia-vascular.com/aneurismas/ [Accedido: 30-may-2018].

[22] "Waku Planet". [En línea]. Disponible en: http://wakuplanet.com/580rejilla-ajustable-para-ventanas.html [Accedido: 30-may-2018].

[23] "Colegio Argentino de Cardioangiólogos intervencionistas". [En línea]. Disponible en: http://caci.org.ar/post/stents [Accedido: 30-may-2018].

[24] Suárez, G. y Bustamante, J. "Diseño y desarrollo de un nuevo anillo de soporte para bioprótesis valvular cardíaca porcina y/o humana". Conf. Gaceta Cardiovascular ISSN:0124-7247 ed: Clínica cardiovascular Santa María, Colombia, 2001, v. fasc. pp. 2-2.

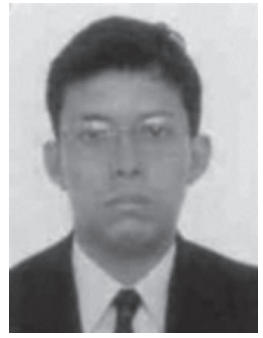

Gustavo Suárez Guerrero es un investigador del Grupo de Investigación en Dinámica Cardiovascular, y Coordinador del grupo de Matemáticas de la Universidad Pontificia Bolivariana. Obtuvo su grado en Ingeniería Mecánica por la Universidad Pontificia Bolivariana, Medellín, Colombia, en 1995. Luego recibió su doctorado en Ingeniería de la Universidad Pontificia Bolivariana, Medellín, Colombia, en 2011, y Doctor en Ciencias Matemáticas por la Universidad Pierre et Marie Curie, París, Francia, en 2012. Sus áreas de interés son los sistemas de biomecánica, modelado y simulación, las matemáticas y los métodos numéricos aplicados. ORCID: https://orcid.org/0000-00017595-7366

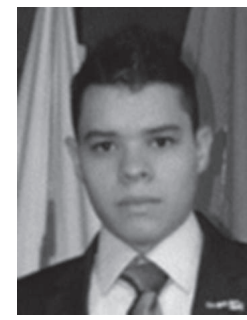

Sebastián Aristizábal Suárez es un investigador del Grupo de Investigación en Dinámica Cardiovascular, y del grupo de Matemáticas de la Universidad Pontificia Bolivariana. Obtuvo su Grado en Ingeniería Biomédica, en 2015. Comenzó sus estudios de maestría en ingeniería con énfasis en biomédica, en 2016. Sus áreas de interés son: Modelación matemática de fenómenos biomédicos. Simulación computacional de fenómenos biomédicos, diseños biomédicos, stent endovasculares. ORCID: https://orcid.org/00000002-3078-1165

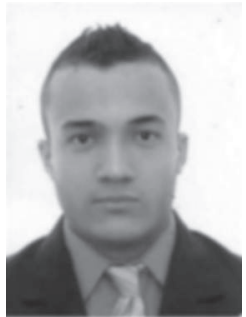

Juan Sebastián Parra Sánchez es un investigador del grupo de Matemáticas de la Universidad Pontificia Bolivariana. Obtuvo su Grado en Ingeniería eléctrica por la Universidad Pontificia Bolivariana, Medellín, Colombia. Sus áreas de interés son: Modelación matemática de fenómenos de ingeniería, Simulación computacional de fenómenos de ingeniería y métodos numéricos. 